Revista Brasileira de Agricultura Irrigada v.13, no.3, p. 3402 - 3411, 2019

ISSN 1982-7679 (On-line)

Fortaleza, CE, INOVAGRI - http://www.inovagri.org.br

DOI: $10.7127 /$ rbai.v13n301009

Protocolo 1009.19 - 31/08/2018 Aprovado em 27/06/2019

\title{
TOMATE SWEET GRAPE CULTIVADO COM DIFERENTES LÂMINAS E FREQUENCIAS DE IRRIGAÇÃO EM AMBIENTE PROTEGIDO
}

\author{
Ewerton Dilelis Ferreira ${ }^{1}$, Miguel Augusto Viol $^{2}$, Jacinto de Assunção carvalho ${ }^{3}$, Mariana Lúcio \\ Gontijo $^{4}$, Fátima Conceição Rezende ${ }^{5}$, Elvis Marcio de Castro Lima ${ }^{6}$
}

\begin{abstract}
RESUMO
Atualmente os tomates do grupo grape estão ocupando maior espaço no mercado consumidor brasileiro. Entretanto, algumas informações relativas ao seu manejo, como a quantidade de água que melhor atenda às necessidades da cultura durante o seu ciclo ainda são escassas. Assim, o objetivo deste trabalho foi avaliar o efeito de diferentes lâminas e frequências de irrigação sobre as características produtivas do tomate sweet grape cultivado em ambiente protegido. As plantas foram conduzidas em vasos e irrigadas por gotejamento. Utilizou-se o delineamento em esquema fatorial com cinco lâminas $(40,60,100,120$ e 140\% de reposição do minitanque) por três frequências $(1,2$ e 3 irrigações ao dia), com quatro repetições. Foram avaliados o número, a produtividade, o diâmetro e o comprimento dos frutos e a eficiência de uso da água (EUA). Todos os atributos avaliados foram significativamente influenciados pela lâmina de irrigação apresentando um ajuste linear positivo. $\mathrm{O}$ maior valor de EUA foi obtido com irrigação com base na reposição de $60 \%$ da evaporação do tanque classe A.
\end{abstract}

Palavras-chave: gotejamento, mini tomate, eficiência de uso da água

\section{SWEET GRAPE TOMATO GROWN WITH DIFFERENT DEPTHS AND FREQUENCIES OF IRRIGATION IN PROTECTED ENVIRONMENT}

\footnotetext{
ABSTRACT

1 Aluno do curso de Agronomia Universidade Federal de Lavras, Lavras, Minas Gerais, Brasil. Email: ewertondilelis@yahoo.com.br

${ }^{2}$ Aluno do curso de Agronomia Universidade Federal de Lavras, Lavras, Minas Gerais, Brasil. gutoviol@hotmail.com

${ }^{3}$ Prof. Titular DSC Dep. Recursos Hídricos e Saneamento da Universidade Federal de Lavras, Lavras, Minas Gerais, Brasil. E-mail:jacintoc@ufla.br

${ }^{4}$ Doutoranda em Recursos Hídricos em Sistemas Agrícolas, Universidade Federal de Lavras, Lavras, Minas Gerais, Brasil. E-mail:mariana.gontijo@ufv.br

5 Eng. Agríc. DS em Irrigação e Drenagem da Universidade Federal de Lavras, Lavras, Minas Gerais, Brasil. Email:frezende@ufla.br

${ }^{6}$ Eng. Agríc. DS em Recursos Hídricos em Sistemas Agrícolas da Universidade Federal de Lavras, Lavras, Minas Gerais, Brasil. E-mail:frezende@ufla.br
} 
Sweet grape tomatoes are increasing participation in the Brazilian market. However, information about crop management, such as water requirements during the crop is still scarce. Thus, this paper aimed to evaluate the effect of different irrigation depths and frequencies on the productive characteristics of sweet grape tomato cultivated in greenhouse production. The crop was grown in pots and irrigated by drip. We used a statistical factorial scheme design with five irrigation water depths $(40,60,100,120$ and $140 \%$ of replacement measured with a reduced pan) with three frequencies (1, 2 and 3 irrigations per day) and four replications. The number, productivity, diameter and fruits' length and water use efficiency (WUE) were evaluated. All evaluated attributes were statistically significantly influenced by the irrigation depths with a positive linear fit. The highest WUE value was obtained with irrigation based on the $60 \%$ replacement of the reduced pan's evaporation.

Keywords: drip, mini tomato, water use efficiency

\section{INTRODUÇÃO}

A produção nacional de tomate em 2016 foi superior a 3,6 milhões de toneladas, no qual se destaca a região sudeste com aproximadamente 1,8 milhões de toneladas (IBGE, 2017). Estes dados evidenciam a importância da cultura do tomate no agronegócio brasileiro, com destaque para a região sudeste. Dentre os grupos de tomate há aqueles classificados como mini, destacando entre eles o tomate cereja e tomate grape. Atualmente os tomates grape estão ocupando espaço no mercado, tendo como seu maior diferencial o elevado grau brix (doçura) e praticidades. As cultivares desse tipo de tomate produzem frutos com massa média de doze gramas, de cor vermelho intenso e com formato semelhante ao de uma baga de uva (ALVARENGA, 2013).

A cultura do tomate é exigente em água e o uso eficiente da água na agricultura é uma meta que deve ser alcançada pelos produtores uma vez que, a água é um recurso natural não renovável e, também, devido à irregularidade no regime pluviométrico. Dessa forma o uso racional da água na agricultura deve ser prioridade e a adoção de técnicas de manejo de irrigação pode permitir economia de água com manutenção de níveis de produtividade satisfatórios (PATANÈ et al., 2011).

Os minis tomates geralmente são cultivados em ambiente protegido, diretamente no solo ou em vasos preenchidos com substratos comerciais. Estudos realizados por Cunha et al. (2014) com o mini tomate sweet grape cultivado com diferentes tipos de substrato e solução nutritiva indicou que o tipo de substrato e a interação substrato $\mathrm{x}$ solução nutritiva influenciaram significativamente no número total de frutos. $\mathrm{O}$ sistema de condução das plantas e o tipo de poda de mini tomate, também podem influenciar na produtividade conforme foi observado por foi Takahashi e Cardoso (2015).

Entretanto para que a produtividade das cultivares seja maximizada é necessário fazer manejo criterioso da irrigação e da aplicação de fertilizantes. Abrahão et al. (2011), avaliaram o efeito de diferentes relações entre $\mathrm{K}: \mathrm{Ca}: \mathrm{Mg}$ em solução nutritiva na produção de mini tomate sweet million e sweet grape cultivados em substratos e verificaram que as soluções não influenciaram na produção. $\mathrm{Na}$ avaliação de diferentes lâminas e frequências de irrigação em tomate cereja sweet million, cultivado em ambiente protegido Franca et al. (2017), verificaram que a frequência de irrigação não influenciou estatisticamente na produtividade $\mathrm{e}$ que a maior produtividade foi obtida no tratamento irrigado com lâmina equivalente a $75 \%$ da evapotranspiração da cultura (ETC).

Resultados de pesquisa sobre manejo de irrigação no cultivo de mini tomate ainda são escassos, assim, objetivou-se com este trabalho avaliar o efeito de diferentes lâminas de irrigação e frequência de aplicação nas características produtivas do mini tomate sweet grape cultivado em vasos em ambiente protegido.

\section{MATERIAL E MÉTODOS}

O experimento foi conduzido em casa de vegetação localizada na Universidade Federal de Lavras, na região do Sul do Estado de Minas 


\section{TOMATE SWEET GRAPE CULTIVADO COM DIFERENTES LÂMINAS E FREQUÊNCIAS DE IRRIGAÇÃO EM AMBIENTE PROTEGIDO}

Gerais, a $896 \mathrm{~m}$ de altitude, $21^{\circ} 13^{\prime} 55^{\prime}$ ' de Latitude Sul e $44^{\circ} 58^{\prime}$ '34', de Longitude Oeste. Segundo a classificação de Koppen a região apresenta um clima Cwa, ou seja, clima temperado suave, chuvoso, com inverno seco, temperatura média dos 3 meses mais frio inferior a $18^{\circ} \mathrm{C}$ e superior a $3^{\circ} \mathrm{C}$, o verão apresenta temperaturas médias que variam de $22,1{ }^{\circ} \mathrm{C}$ em março a $22,8{ }^{\circ} \mathrm{C}$ em fevereiro. A precipitação anual média é de $1460 \mathrm{~mm}$ (DANTAS et al., 2007).

A casa de vegetação foi construída com estrutura metálica, cobertura tipo arco, com dimensões de 30,0 $\mathrm{m}$ de comprimento, 7,0 $\mathrm{m}$ de largura e pé-direito de $4,0 \mathrm{~m}$. A mesma foi coberta com filme de polietileno transparente, espessura de 150 micras, com tratamento antiUV. As fachadas laterais e frontais foram fechadas com tela anti-afídica.

A evaporação no interior do ambiente foi medida em um minitanque $(0,60 \mathrm{~m}$ de diâmetro e $0,25 \mathrm{~m}$ de altura) instalado no centro do ambiente. Foi colocado um termohigrômetro a $1,5 \mathrm{~m}$ de altura para monitoramento das temperaturas e umidades relativas do ar. Os dados de temperatura (máxima, mínima e atual) e umidade relativa do ar (máxima, mínima e atual) bem como a leitura do minitanque era realizado diariamente às 9 horas.

Utilizou-se o delineamento em esquema fatorial de $5 \times 3$ com quatro repetições. Os tratamentos consistiram de cinco níveis de reposição da lâmina evaporada no minitanque (E) definidas por 40, 60, 100, 120 e $140 \%$, e três frequências de irrigação (F) definidas por 1,2 e 3 vezes ao dia, bem como a interação entre os dois fatores $(\mathrm{E} \times \mathrm{F})$.

As plantas foram transplantadas em vasos de polietileno com volume total de 8 litros, com uma planta por vaso. Os vasos foram preenchidos com solo, classificado como Latossolo Vermelho Distroférrico (LVd) de textura argilosa (Areia 9\%, Silte $24 \%$ e Argila $67 \%$ ). O solo foi coletado na camada superficial (0 a $20 \mathrm{~cm}$ de profundidade). Após a coleta, o solo foi secado e passado em peneira de $4 \mathrm{~mm}$ para redução dos torrões.

A adubação inicial (de plantio) para os vasos com solo foi realizada com base nos resultados da análise de fertilidade do solo e de acordo com as recomendações da $5^{\text {a }}$ Aproximação da Comissão de Fertilidade do Solo do Estado de Minas Gerais (CFSEMIG, 1999). As adubações suplementares foram realizadas via fertirrigação, de acordo com a recomendação para cultura e conforme correções recomendadas pela empresa responsável pelo material genético (Sakata Seed Sudamerica).

As mudas foram formadas pelo Grupo Agro e transplantadas 45 dias após a semeadura, apresentando duas guias conforme adotado para esse tipo de cultivar. O espaçamento entre linhas de plantio foi $1,10 \mathrm{~m}$ e entre plantas de $0,30 \mathrm{~m}$, colocando uma muda por vaso.

O tomate Sweet Grape foi conduzido verticalmente, tutorado com fitilhos. Foi retirado o ápice caulinar quando a planta atingiu altura igual ou superior a $2,10 \mathrm{~m}$. $\mathrm{O}$ espaçamento entre moirões na linha de plantio foi 3,0 m. Cada moirão era composto por uma trava individual, colocada na parte superior e medindo $0,75 \mathrm{~m}$. Nas extremidades das travas foram passados fios de arame liso para sustentação das plantas, amarrando-se fitilhos em uma da extremidade da planta e a outra no arame.

Os tratos culturais consistiram em retirada de brotos e no enrolamento das plantas no fitilho. A retirada de brotações foi realizada semanalmente. O controle fitossanitário foi realizado conforme a necessidade do uso de inseticidas e fungicidas, sempre respeitando o período de carência de cada produto.

O sistema de irrigação foi composto por válvulas solenoides, sistema de bombeamento, painel controlador, injetor de fertilizantes, mangueiras de PEBD e gotejadores com vazão nominal de $2,11 \mathrm{~L} \mathrm{~h}^{-1}$. Foi utilizado um gotejador por planta. A irrigação foi realizada diariamente e a lâmina aplicada foi calculada com base na evaporação do minitanque instalado dentro do ambiente. Para garantir o pegamento das mudas, todas as plantas receberam 21,84 mm de água via irrigação nos primeiros 12 dias após transplantio (DAT). O experimento foi conduzido por 120 dias após 
o transplantio. Foram colhidos os frutos quando atingiram o ponto de maturação com coloração avermelhada em toda superfície do fruto. Foi avaliado o peso dos frutos por planta e número de frutos, diâmetro e comprimento dos frutos. E a eficiência de uso da água. Os frutos foram pesados em balança digital e medidos o diâmetro e o comprimento com paquímetro digital. A eficiência do uso da água foi obtida pela relação entre o peso de frutos por planta e a lâmina de água aplicada em cada tratamento. Foi realizado o teste de Kolmogorov-Smirnov para o erro experimental, com objetivo de verificar a normalidade dos dados das variáveis analisadas (Tabela 1).

Observa-se que todas as variáveis analisadas apresentaram distribuição normal. Posteriormente, foi realizado à análise de variância dos dados com aplicação do teste $\mathrm{F}$ ao nível de $1 \%$ e $5 \%$ de significância e, quando significativo, realizou a análise de regressão.

As análises estatísticas foram realizadas utilizando-se o software SISVAR versão 5.3 (FERREIRA, 2011).

Tabela 1. Teste de Kolmogorov-Smirnov para as variáveis analisadas no cultivo do tomate Sweet Grape.

\begin{tabular}{cccc}
\hline Variáveis & K-S calculado & K-S Tabelado 5\% & Resultado \\
\hline Número de frutos & 0,06691 & 0,157 & $\mathrm{~ns}$ \\
Diâmetro dos frutos & 0,08426 & 0,157 & $\mathrm{~ns}$ \\
Comprimento dos frutos & 0,07620 & 0,157 & $\mathrm{~ns}$ \\
Produtividade & 0,09114 & 0,157 & $\mathrm{~ns}$ \\
Eficiência de uso da água & 0,09608 & 0,157 & $\mathrm{~ns}$ \\
\hline
\end{tabular}

\section{RESULTADOS E DISCUSSÃO}

O total de água aplicado em volume e lâminas de irrigação durante o período de condução do experimento em cada tratamento estão apresentadas na Tabela 2. Os tratamentos foram diferenciados a partir
16/04/2016, 13 dias após o transplantio das mudas, onde foi conduzido até o dia 01/08/2016. Nos primeiros 12 DAT, período de pegamento das plantas, a lâmina aplicada diariamente foi em média $1,82 \mathrm{~mm}$.

Tabela 2. Volume de água e lâminas de água totais aplicadas durante a condução do tomate sweet grape, correspondente a cada percentual da evaporação do minitanque.

\begin{tabular}{ccc}
\hline Tratamentos $(\%)$ & Volume aplicado $(\mathrm{L})$ & Lâmina aplicada $(\mathrm{mm})$ \\
\hline 40 & 20,4 & 56,7 \\
60 & 30,6 & 85,0 \\
100 & 50,9 & 141,1 \\
120 & 61,0 & 169,5 \\
140 & 71,3 & 198,1 \\
\hline
\end{tabular}

O resultado da análise de variância das características de produção do mini tomate estão apresentados na Tabela 3. Verifica-se que as lâminas de irrigação aplicadas nos diferentes níveis da evaporação do minitanque teve efeito significativo sobre todas as características avaliadas. Entretanto, para a interação (E x F) e para a frequência de irrigação não houve efeito significativo para nenhuma das características avaliadas. 


\section{TOMATE SWEET GRAPE CULTIVADO COM DIFERENTES LÂMINAS E FREQUÊNCIAS DE IRRIGAÇÃO EM AMBIENTE PROTEGIDO}

Tabela 3. Resumo da análise de variância para número de frutos (NF), diâmetro (D) e comprimento (C) de frutos, produtividade média (P) e eficiência de uso da água (EUA) do tomate sweet grape cultivados em vaso e em ambiente protegido.

\begin{tabular}{ccccccc}
\hline Tratamento & GL & \multicolumn{5}{c}{ Quadrados Médios } \\
\cline { 3 - 7 } & & $\mathrm{NF}$ & $\mathrm{D}(\mathrm{mm})$ & $\mathrm{C}(\mathrm{mm})$ & $\mathrm{P}\left(\mathrm{g} \mathrm{planta}^{-1}\right)$ & $\mathrm{EUA}\left(\mathrm{g} \mathrm{mm}^{-1}\right)$ \\
Evaporação(\%) & 4 & $293622,50^{*}$ & $12,47^{*}$ & $51,014^{*}$ & $1143286,62^{*}$ & $3,42^{\mathrm{ns}}$ \\
Frequência(F) & 2 & $833,29^{\text {ns }}$ & $0,80^{\mathrm{ns}}$ & $5,70^{\mathrm{ns}}$ & $26742,58^{\mathrm{ns}}$ & $2,38^{\text {ns }}$ \\
E x F & 8 & $982,89^{\mathrm{ns}}$ & $0,70^{\mathrm{ns}}$ & $1,00^{\mathrm{ns}}$ & $15061,44^{\mathrm{ns}}$ & $0,83^{\text {ns }}$ \\
Erro & 60 & 15154,93 & 0,93 & 2,01 & 30053,20 & 2,21 \\
Total & 74 & & & & & \\
CV & & 28,15 & 4,98 & 4,90 & 24,95 & 27,33 \\
Média & & 138,29 & 19,44 & 2,99 & 694,77 & 5,45 \\
\hline
\end{tabular}

*significativo a $1 \%$ de significância e ns não significativo pelo teste $\mathrm{F}$

No trabalho de Franca et al. (2017) com tomate cereja, também foi observado que as lâminas de irrigação utilizadas, aplicadas com frequência de 1,2 e 3 vezes ao dia, não influenciaram na produtividade e que não houve interação entre lâmina e frequência de irrigação. $\mathrm{O}$ número de frutos aumentou linearmente com o aumento do percentual da evaporação do minitanque pode ser observado na Figura 1.

Nota-se, que o ajuste linear descreve o processo com coeficiente de determinação de $95,74 \%$, e que o aumento de uma unidade no percentual da evaporação do minitanque promove o incremento no número de frutos da ordem de 1,0435 .

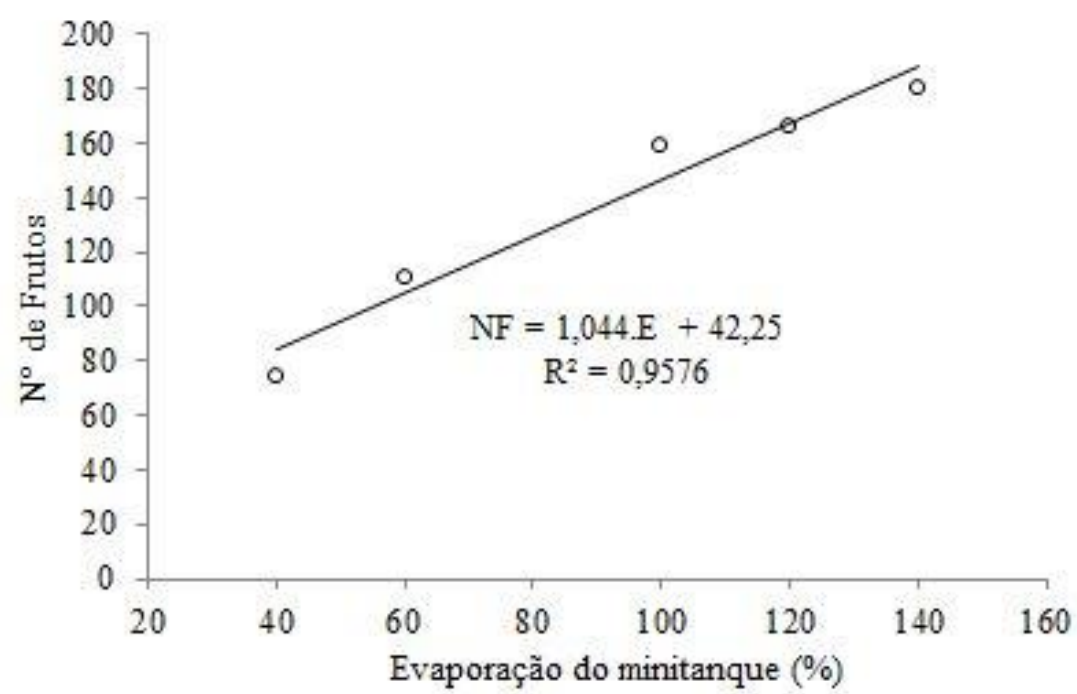

Figura 1. Número de frutos do tomate sweet grape por planta em função do percentual de evaporação do minitanque

O menor número médio de frutos $(74,6)$ foi obtido no tratamento irrigado com lâmina de $56,7 \mathrm{~mm}(40 \%)$ e o maior número de frutos $(180,6)$ foi registrado na lâmina de 198,1 $\mathrm{mm}(140 \%)$, ou seja, um acréscimo da ordem de $142 \%$. Para obter este aumento no número de frutos foi necessário aumentar a aplicação de água em $249,4 \%$. Avaliando as características produtivas de diferentes genótipos de mini tomate, Preczenhak et al. (2014), obtiveram para a cultivar sweet grape um número médio de 145 frutos por planta. Valor semelhante foi obtido por Abrahão et al. (2011) para o tomate sweet grape. Uma média de 136,8 frutos por 
planta foi obtido por Rocha et al. (2010), avaliando o efeito da concentração iônica e da posição do cacho floral de tomate cereja cultivado em ambiente protegido. Avaliando o efeito da poda apical e da condução de haste de tomate sweet grape, Takahashi e Cardoso (2015), verificaram que a condução de haste teve efeito significativo no número de frutos produzidos. A forma de condução das plantas (número de cachos, poda apical, retirada de brotos, etc.) são fatores que podem influenciar no número de frutos, bem como o manejo da irrigação. Observa-se que o maior número de frutos foi obtido com a maior lâmina aplicada, entretanto há indicativos de que uma lâmina maior pode aumentar o número de frutos.

O diâmetro do fruto também apresentou um ajuste linear em função do percentual da evaporação do minitanque (Figura 2), neste, verificou-se um incremento de $0,0156 \mathrm{~mm}$ no diâmetro dos frutos com o aumento da unidade do nível da evaporação do minitanque, com valores médios variando de $18,17 \mathrm{~mm}(40 \%)$ a $20,53 \mathrm{~mm}(140 \%)$. Trabalhando com o tomate sweet grape, Preczenhak et al. (2014), obteve valor de diâmetro de 26,14 mm. Trabalho realizado por Candian et al. (2015), em que avaliaram o efeito do sistema de condução da haste em mini tomate (híbrido Coco) com manejo orgânico foi registrado valores de diâmetro de fruto variando de $31,06 \mathrm{~mm}$ a $33,31 \mathrm{~mm}$. A diferença do diâmetro de fruto observada entre os dois trabalhos pode ser devido à cultivar utilizada bem como a fatores relacionados ao sistema de condução das plantas dos experimentos.

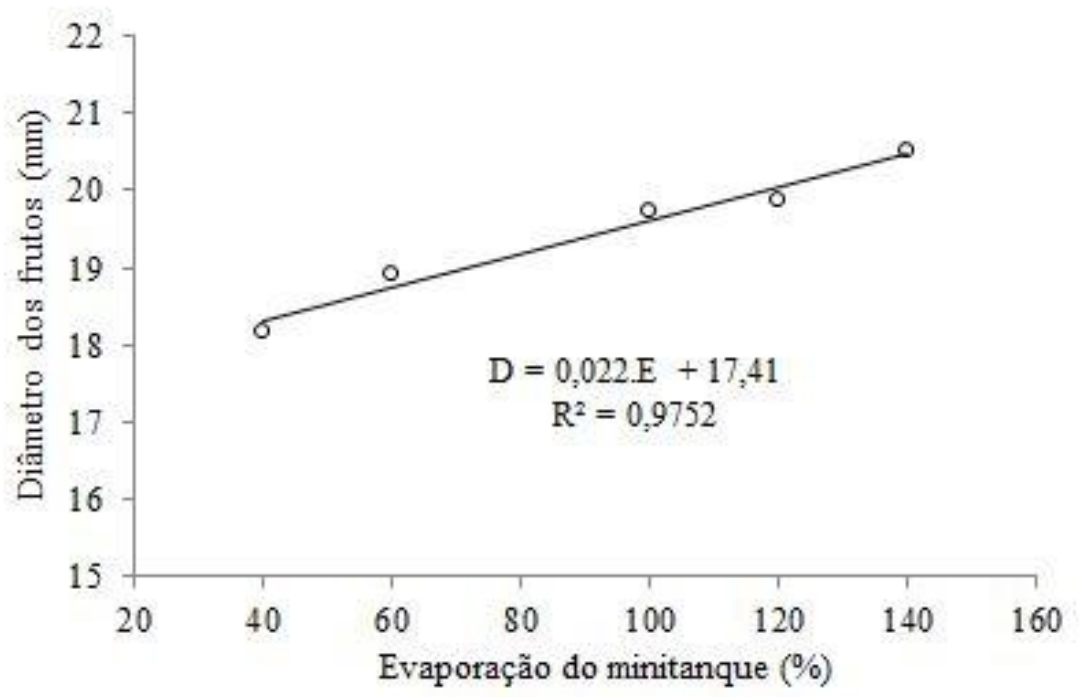

Figura 2. Diâmetro médio de fruto do tomate sweet grape em função do percentual da evaporação do minitanque

O comprimento médio do fruto variou de $26,43 \mathrm{~mm}$ (40\% da evaporação do minitanque) a $30,9 \mathrm{~mm} \quad(140 \%$ da evaporação do minitanque), (Figura 3). Nota-se, que o ajuste linear descreve o processo com coeficiente de determinação de $98,05 \%$, e que o aumento de uma unidade do percentual da evaporação do minitanque promove o incremento no comprimento médio de frutos da ordem de 0,0313 . 


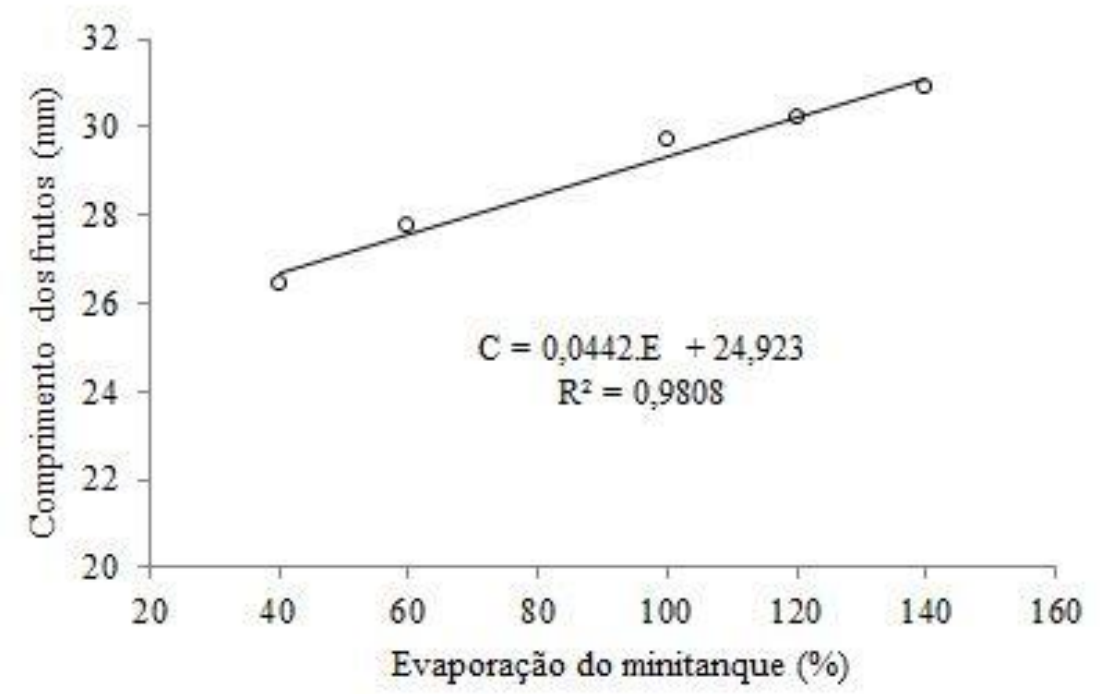

Figura 3. Comprimento médio de fruto do tomate sweet grape em função do percentual da evaporação do minitanque

O comprimento do fruto ou diâmetro longitudinal é um dos atributos utilizados para classificação do tomate e de acordo com a classificação do tomate proposto pelo BRASIL (1995), os minis tomates não se enquadram nas normas de classificação para o atributo tamanho de frutos. A produtividade aumentou linearmente com o aumento do percentual da evaporação do minitanque, variando de 307,03 a 990,40 g planta $^{-1}$. Observa-se, através da equação ajustada, que há um aumento no peso médio do fruto de $4,659 \mathrm{~g}$ com o aumento na unidade do percentual da evaporação do minitanque (Figura 4).

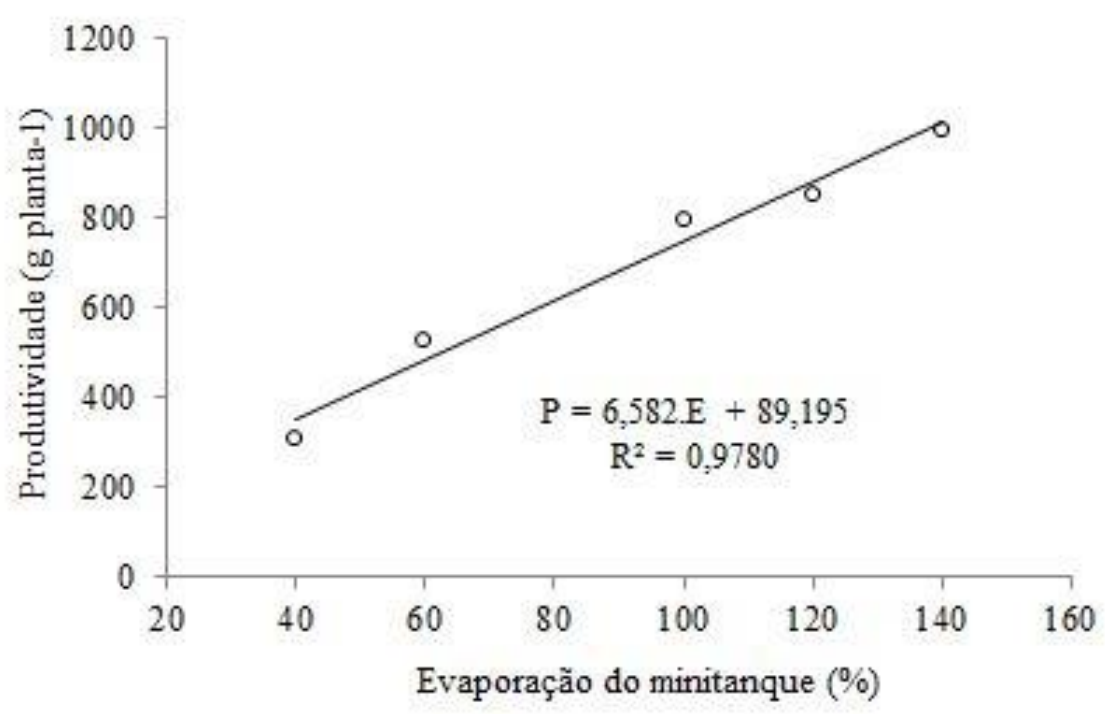

Figura 4. Produtividade do tomate sweet grape em função do percentual da evaporação do minitanque

A diferença no peso médio de frutos por planta, entre a maior e menor de lâmina aplicada, foi de aproximadamente $222 \%$. Abrahão et al. (2011), trabalhando com a cultivar sweet grape, obteve produtividade de
$1300 \mathrm{~g}$ planta $^{-1}$ e peso médio de frutos de $9,0 \mathrm{~g}$. Já no trabalho de Preczenhak et al. (2014) a produtividade foi de $2078,92 \mathrm{~g}_{\text {planta }}{ }^{-1}$ e peso médio de frutos de 14,48 g. As diferenças dos valores de produtividade pode ser devido ao 
número de colheitas realizadas, manejo da cultura bem como a fatores ambientais (Temperatura, umidade relativa e radiação, etc), predominantes nos locais onde foram desenvolvidos os experimentos.

Os valores médios da eficiência de uso da água são apresentados na Figura 5. Observa-se que o maior valor de EUA foi obtido no tratamento irrigado com a lâmina de $85 \mathrm{~mm}$, correspondente a $60 \%$ da lâmina de evaporação do minitanque. No tratamento irrigado com lâmina de 56,7 mm (40\%) o déficit hídrico pode ter induzido a um estresse mais severo promovendo a redução na produção de frutos.

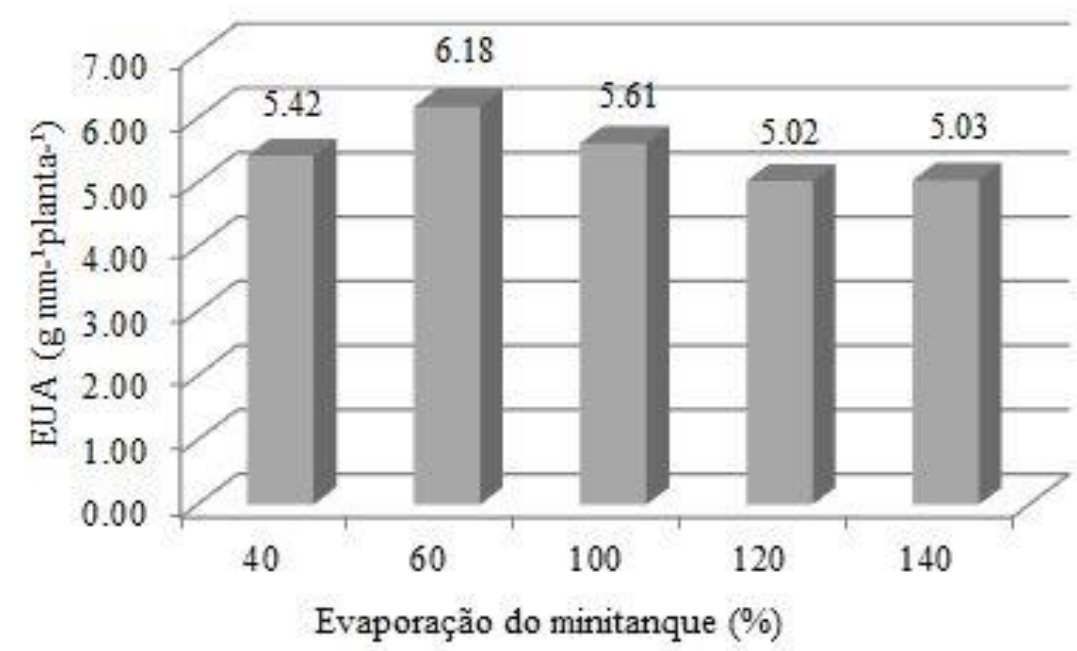

Figura 5. Eficiência do uso da água (EUA) do tomate sweet grape em função do percentual da evaporação do minitanque

A resposta da eficiência de uso da água à disponibilidade hídrica depende do nível de estresse hídrico experimentado pelas culturas (CANTORE et al., 2016). Em condições de déficit hídrico moderado, ocorre o fechamento parcial dos estômatos e a transpiração reduz mais do que a fotossíntese e em consequência a eficiência de uso da água aumenta (FAVATTI et al., 2009; PATANÉ et al., 2011; CHEN et al., 2013). A eficiência do uso da água aumenta com o déficit hídrico, porém, quando foi calculada com base na produtividade comercial o maior valor não foi registrado no tratamento não irrigado, uma vez que o déficit hídrico severo aumenta a produtividade não comercial (PATANÈ et al., 2011).

\section{CONCLUSÕES}

O potencial produtivo do tomate sweet grape foi afetado pelas lâminas de irrigação aplicadas neste estudo.

A frequência de irrigação não afetou significativamente o número de frutos por planta, produtividade dos frutos e eficiência de uso da água, de modo que uma irrigação por dia por ser realizada sem prejuízo na produção do tomate sweet grape.

\section{REFERÊNCIAS BIBLIOGRÁFICAS}

ABRAHÃO, C.; VILLAS BÔAS, R. L.; SILVA, V. de C.; RAMOS, A. R. P.; CAMPAGNOL, R.; BARDAVIESSO, D. M. Produção de mini tomate em função de diferentes relações de $\mathrm{K}: \mathrm{Ca}: \mathrm{Mg}$ na solução nutritiva. Horticultura Brasileira, v. 29, n. 2, p. S3823-S3819, 2011.

ALVARENGA, M. A. R. (Org.). Tomate: produção em campo, em casa de vegetação $\mathrm{e}$ em hidroponia. 2 Edição, Lavras: Editora Universitária de Lavras, 2013, 457p;

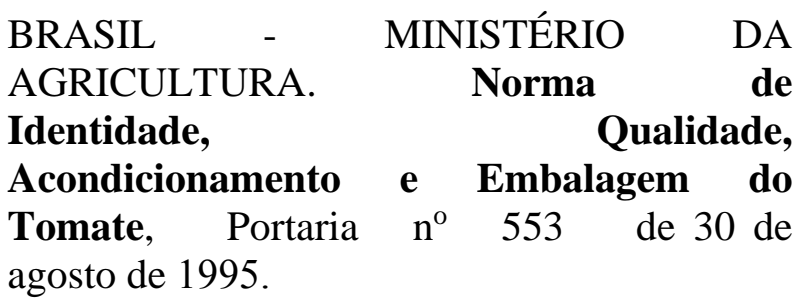




\section{TOMATE SWEET GRAPE CULTIVADO COM DIFERENTES LÂMINAS E FREQUENCIAS DE IRRIGAÇÃO EM AMBIENTE PROTEGIDO}

CANDIAN, J. S.; MARTINS, B. N. M.; CARDOSO, A. I. I.; EVANGELISTA, R. M.; FUJITA, E. Stem conduction systems effect on the production and quality of mini tomato under organic management. Bragantia, v. 76, n. 2, p. 238-245, 2017.2 DOI: http://dx.doi.org/10.1590/1678-4499.558.

CANTORE, V.; LECHKAR, O.; KARABULUT, E.; SELLAMI, M. H.; ALBRIZIO, R.; BOARI, F.; STELLACCI, A. M.; TODOROVIC, M. Combined effect of deficit irrigation and strobilurin application on yield, fruit quality and water use efficiency of "cherry" tomato (Solanum lycopersicum L.). Agricultural Water Management, v. 167. p. 53-61, 2016. https://doi.org/10.1016/j.agwat.2015.12.024.

CHEN, J.; KANG, S.; DU, T.; QJU, R.; GUO, P.; CHEN, R. Quantitative response of greenhouse tomato yield and quality to water deficit at different growth stages. Agricultural Water Management, v. 129, p. 152-162, 2013. https://doi.org/10.1016/j.agwat.2013.07.011.

CFSEMIG - COMISSÃO DE FERTILIDADE DO SOLO DO ESTADO DE MINAS GERAIS. Recomendação para o uso de corretivos e fertilizantes em Minas Gerais: $5^{\text {a }}$ aproximação, Viçosa, MG: UFV, 1999.

CUNHA, A. H. N.; SANDRI, D.; VIEIRA, J. A.; CORTEZ, T. B.; OLIVEIRA, T. H. de. Sweet grape mini tomato grown in culture substrates and effluent with nutrient complementation. Engenharia Agrícola, v. 34, n. 4, p. 707-715, 2014. https://doi.org/10.1590/S0100-

69162014000400010.

DANTAS, A. A. A.; CARVAlHO, L. G.; FERREIRA, E. Classificação e tendência climática em Lavras, MG. Ciência e Agrotecnologia, v. 31, n. 6, p. 1862-1866, 2007.

FAVATTI, F.; LOVELLI, S.; GALGANO, F.; MICCOLIS, V.; DI TOMMASO, T.;
CANDIDO, V. Processing tomato quality as affected by irrigation scheduling. Scientia Horticulturae, v. 122, n. 4, p. 562-571, 2009. https://doi.org/10.1016/j.scienta.2009.06.026

FERREIRA， D.F. SISVAR: Um sistema computacional de análise estatística. Ciência e Agrotecnologia, v.35, p.1039- 1042, 2011. https://doi.org/10.1590/S141370542011000600001.

FRANCA, R. J. da F.; LEITÃO, M. de M. V. B. R.; CAMPECHE, L. F. de S. M. Produtividade do tomate cereja em ambiente protegido e céu aberto em função das lâminas e intermitências de irrigação. Revista Brasileira de Agricultura Irrigada, v. 11, n. 2, p. 13641370 , 2017. https://doi.org/10.7127/rbai.v11n200628.

IBGE - INSTITUTO BRASILEIRO DE GEOGRAFIA E ESTATÍSTICA. Levantamento Sistemático da Produção Agrícola. Tabela 1618. Área plantada, área colhida e produção por ano da safra e produto, $2016 . \quad$ Disponível em: $<$ https://sidra.ibge.gov.br/tabela/1618\#resultad o>. Acesso em: 07 jun. 2017.

PATANÈ, C.; TRINGALI, S.; SORTINO, O. Effects of deficit irrigation on biomass, yield, water productivity and fruit quality of processing tomato under semi-arid Mediterranean climate conditions. Scientia Horticulturae, v. 129, n. 4, p. 590-596, 2011. https://doi.org/10.1016/j.scienta.2011.04.030.

PRECZENHAK, A. P.; RESENDE, J. T. V.; CHAGAS, R. R.; SILVA, P. R.; SCHWARZ, K.; RAFAEL, G. F. Caracterização agronômica de genótipos de minitomate. Horticultura Brasileira, v. 32, n. 3, p. 348-356, 2014. https://doi.org/10.1590/S0102053620140003000018.

ROCHA, M de Q.; PEIL, R. M. N.; COGO, C. $M$. Rendimento do tomate cereja em função do cacho floral e da concentração de nutrientes em hidroponia. Horticultura Brasileira, v. 28, n. 
Ferreira et al.

4, $\quad$ p. $\quad$ 466-471, 2010. sistema orgânico com dois tipos de condução https://doi.org/10.1590/S0102de hastes e poda apical. Horticultura 05362010000400015

TAKAHASHI, K.; CARDOSO, A. I. I. Brasileira, v. 33. n. 4, p. 515-520, 2015. Produção e qualidade de mini tomate em https://doi.org/10.1590/S0102053620150000400018 